\title{
The fixed target experiment for studies of baryonic matter at the Nuclotron (BM@N)
}

\author{
Mikhail Kapishin for the BM@N Collaboration ${ }^{1, a}$ \\ ${ }^{1}$ Joint Institute for Nuclear Research, 141980 Russia, Moscow region, Dubna, Joliot Curie 6
}

\begin{abstract}
BM@N (Baryonic Matter at Nuclotron) is the first experiment to be realized at the accelerator complex of NICA-Nuclotron. The aim of the BM@N experiment is to study interactions of relativistic heavy ion beams with fixed targets. The BM@N setup, results of Monte Carlo simulations, the BM@N experimental program and results of technical runs are presented.
\end{abstract}

\section{Introduction}

Relativistic heavy ion collisions provide an unique opportunity to study nuclear matter at extreme density and temperature. In the collision, nuclear matter is heated up and compressed for a very short period of time. At moderate temperatures, nucleons are excited to baryonic resonances which decay by the emission of mesons. At higher temperatures, also baryon-antibaryon pairs are created. This mixture of baryons, antibaryons and mesons, all strongly interacting particles, is denoted as hadronic matter, or baryonic matter if baryons dominate. If the energy density in the formed fireball is sufficiently large the quark-gluon substructure of nucleons becomes visible. At even higher temperatures or densities hadrons melt, and the constituents, quarks and gluons, form a new phase, the Quark-Gluon Plasma (QGP). At these extreme conditions the following features (amongst others) can be studied: the equation-of-state (EoS) of strongly interacting matter at high temperatures and high net-baryon densities; the microscopic structure of strongly interacting matter in dependence on temperature and baryon density; the in-medium modifications of hadrons which might provide information on the onset of chiral symmetry restoration. Theoretical models, however, predict different characteristics of the created matter. New experimental data with high resolution and statistics are needed in order to disentangle different theoretical predictions [1].

\section{Nuclotron heavy-ion physics program}

The ratio of produced mesons to baryons in the fireball increases with the collision energy. A nucleusnucleus collision at the Nuclotron with kinetic beam energy in the range from 1 to $4.5 \mathrm{GeV}$ per nucleon produces a baryon dominated fireball contrary to higher energies at RHIC or SPS. According to the QGSM transport model calculations [2], at Nuclotron energies the nucleon densities in the collision zone of two gold nuclei exceed the saturation density by a factor of $3 \div 4$. At these densities

a e-mail: kapishin@jinr.ru 
nucleons start to overlap. It is expected that under such extreme conditions partial restoration of chiral symmetry might occur [3-7]. It will reveal in in-medium modification of hadrons, in particular, in collisional broadening and dropping mass of vector mesons decaying into di-leptons which are not much effected by final-state interactions.

The relevant degrees of freedom at Nuclotron energies are first of all nucleons and their excited states followed by light and strange mesons. Also the partonic degrees of freedom might show up in small space-time volumes and leave their traces in final hadronic observables. The focus of experimental studies will be on hadrons with strangeness, which are early produced in the collision and not present in the initial state of two colliding nuclei, as nucleons made up from light (u,d)-quarks. The measured production yields of light and strange mesons, as well as of hyperons and anti-hyperons are shown in fig. 1(left) as a function of the nucleon-nucleon collision energy in c.m.s. The Nuclotron beam energy range corresponds to $\sqrt{s_{N N}}=2.3 \div 3.5 \mathrm{GeV}$. It is well suited for studies of strange mesons and multi-strange hyperons produced in nucleus-nucleus collisions close to the kinematic threshold. These studies and the measurements of collective flows of hadrons provide insights on the equation-of-state (EoS) of strongly interacting matter.

Heavy-ion collisions are a rich source of strangeness, and the coalescence of lambda-hyperons with nucleons can produce a variety of light hyper-nuclei $[6,7]$. The study of the hyper-nuclei production is expected to provide new insights into the properties of the hyperon-nucleon and hyperon-hyperon interactions. Figure 1(right) presents the yields of hyper-nuclei as a function of the nucleon-nucleon collision energy in c.m.s. in Au+Au collisions, predicted by a thermal model [9]. The maximum in the hyper-nuclei production rate is predicted at $\sqrt{s_{N N}} \sim 4 \div 5 \mathrm{GeV}$, which is close to the Nuclotron energy range.

In sum, the research program on heavy-ion collisions at the Nuclotron $[1,10]$ includes the following topics: investigation of the reaction dynamics and nuclear EoS, study of the in-medium properties of hadrons, production of (multi)-strange hyperons at the threshold and search for hyper-nuclei. In order to interpret experimental data from heavy-ion collisions and to provide a normalization for the measured $A+A$ spectra, a study of elementary reactions $(p+p, p+n(d))$ is planned.

\section{Detector for studies of Baryonic Matter at Nuclotron (BM@N)}

BM@N (Baryonic Matter at Nuclotron) is the first experiment at the accelerator complex of NICANuclotron. The schematic view of the NICA-Nuclotron complex and the position of the BM@N setup are presented in fig. 2. The sources of light and heavy ions, the beam Booster, Nuclotron accelerator and NICA collider are shown. The heavy-ion physics program of the NICA accelerator complex and the MPD experiment planned at the NICA collider are described in [11-15]. The aim of the $\mathrm{BM} @ \mathrm{~N}$ experiment is to study interactions of relativistic heavy ion beams with fixed targets [10]. The Nuclotron will provide variety of beams from protons to gold ions with the kinetic energy of ions ranging from 1 to $6 \mathrm{GeV}$ per nucleon. The maximum kinetic energy for ions with the ratio of the charge to the atomic weight $(\mathrm{Z} / \mathrm{A})$ of $1 / 2$ is $6 \mathrm{GeV}$ per nucleon. The maximum kinetic energy for gold ions with the ratio of $\mathrm{Z} / \mathrm{A} \sim 1 / 3$ is $4.5 \mathrm{GeV}$ per nucleon. The maximum kinetic energy of protons is 13 $\mathrm{GeV}$. The existing beam line between the Nuclotron and the BM@N experiment is around 160 meter in length. It comprises 26 elements of magnetic optics: 8 dipole magnets and 18 quadruple lenses. An upgrade program of the beam line is foreseen to minimize the amount of scattering material on the way of heavy ions to the BM@N setup.

The planned intensity of the gold ion beam accelerated and accumulated in the Nuclotron and the Booster and transported to the BM@N experimental zone is up to $10^{7}$ ions per second. The gold ion beam is expected in the beginning of 2020. In the period before 2018 the following ions are foreseen 

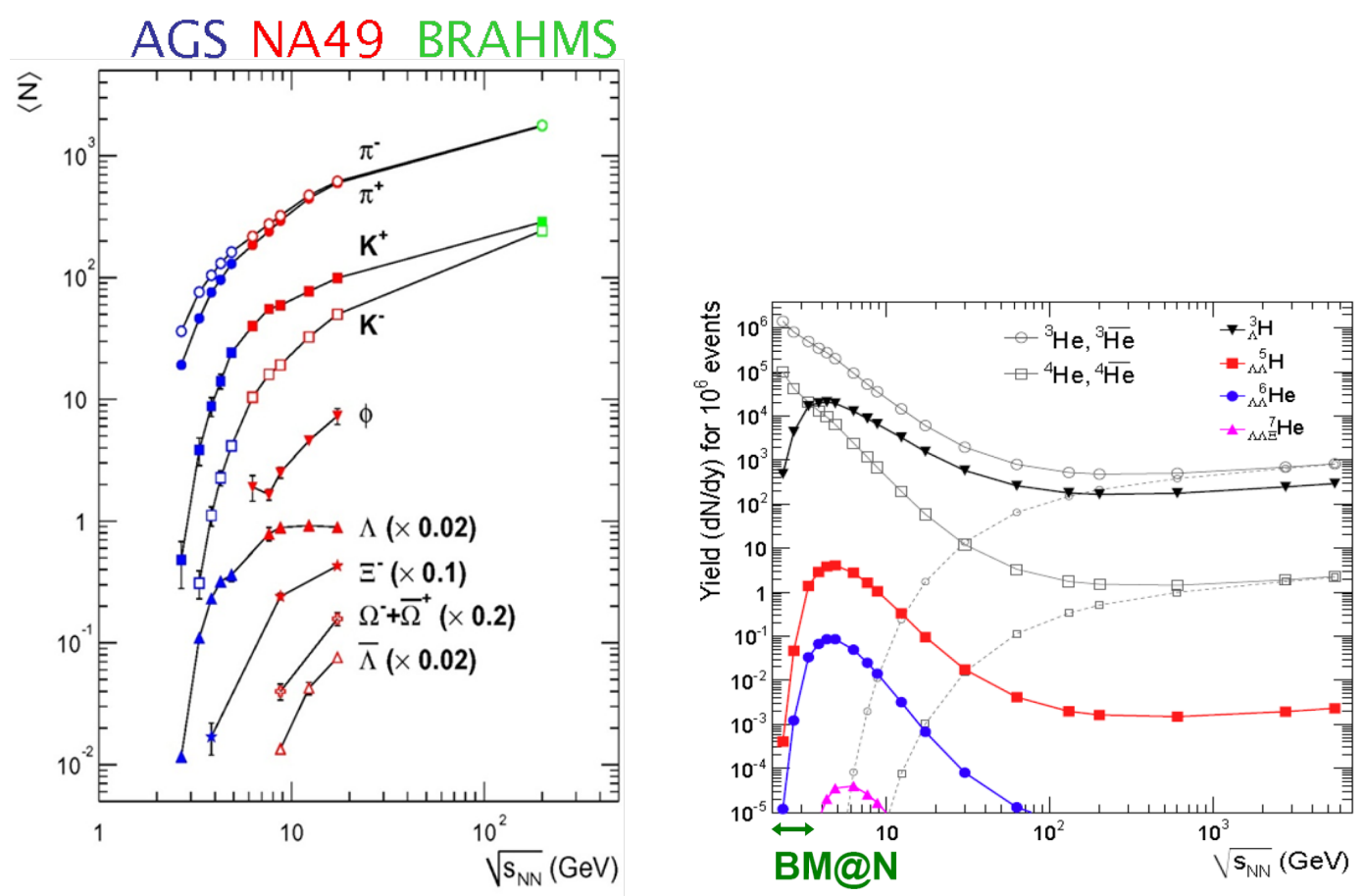

Figure 1. Left plot: Yields of mesons and (anti-) hyperons as a function of the nucleon- nucleon collision energy in c.m.s. in $\mathrm{Au}+\mathrm{Au} / \mathrm{Pb}+\mathrm{Pb}$ collisions, taken from [8]. The Nuclotron BM@ $\mathrm{N}$ beam energy range corresponds to $\sqrt{s_{N N}}=2.3 \div 3.5 \mathrm{GeV}$. Right plot: Yields of hyper-nuclei as a function of the nucleon-nucleon collision energy in c.m.s. in $\mathrm{Au}+\mathrm{Au}$ collisions, calculated with a thermal model [9]. The predicted yields of ${ }^{3} \mathrm{He}$ and ${ }^{4} \mathrm{He}$ nuclei are included for comparison. The Nuclotron BM@N energy range is specified.

to accelerate: the polarized deuteron beam in 2016, the carbon, argon and krypton beams in 2017. In this period of operation the maximal intensity of the beam interacting with the target inside the $\mathrm{BM} @ \mathrm{~N}$ setup is $5 \cdot 10^{5}$ ions per second. The proton-proton interactions will be studied after the realization of the Nuclotron upgrade planned in 2019 using the proton beam and the liquid hydrogen target. Beam types and intensities are specified in table 1.

Figure 3 shows the diagram of the interaction rates accepted by data acquisition systems of heavy ion experiments running at different energies of colliding nuclei. The beam energy range in the BM@N experiment overlaps partially with that in the HADES experiment. The interaction rate of triggered non-peripheral central and intermediate collisions at the second stage of the BM@N experiment is expected to be up to $50 \mathrm{kHz}$. It is limited by the capacity of the readout electronics and data acquisition system. The second stage of the experiment will be realized in 2020 and later.

The minimal configuration of the central tracker in 2017 is based on 6 GEM detectors (half-planes) installed along the beam line. The central tracker will be extended step by step up to eight GEM planes of the full size in beginning of 2020. The factual realization depends on the production capacity of the 


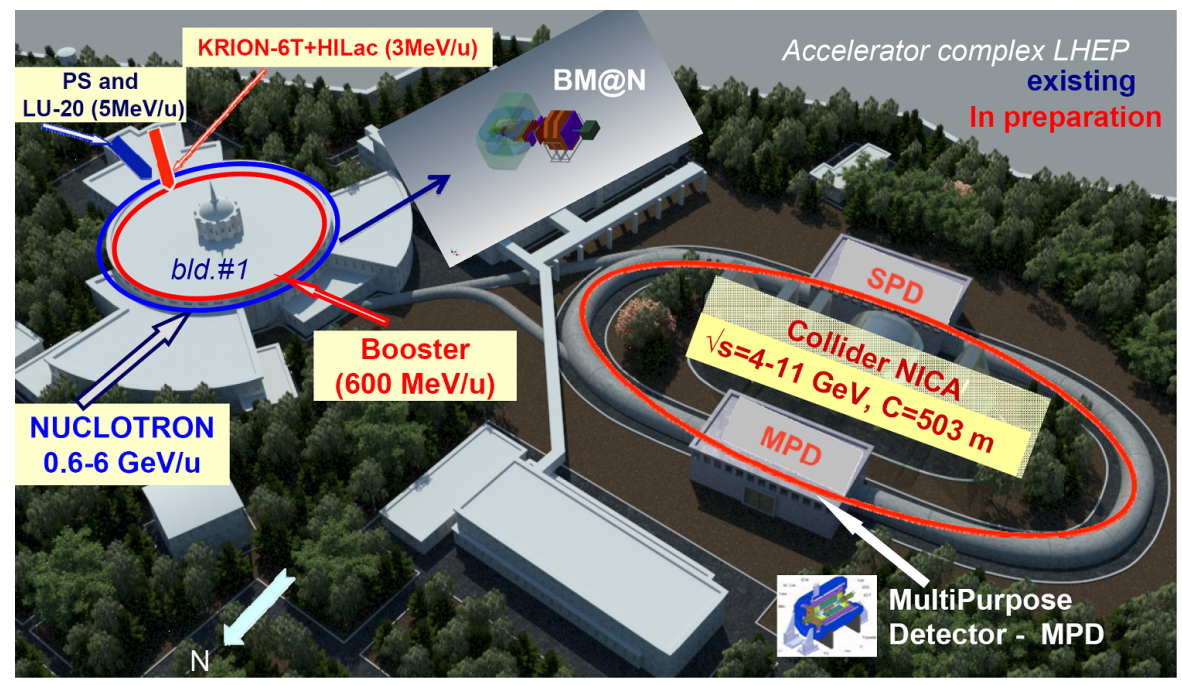

Figure 2. Schematic view of the NICA-Nuclotron complex and the existing position of the BM@N setup.

CERN workshop. The full configuration of the central tracker assumes 8 GEM planes and 4 planes of two-coordinate silicon strip detectors installed in front of the GEM planes to improve the track reconstruction efficiency in $\mathrm{Au}+\mathrm{Au}$ collisions. The central tracker configurations at different stages of the experiment are specified in table 1. Presently, the detectors of this type are being developed for the CBM experiment [17]. The factual realization of the upgrade depends on the time schedule of the silicon tracker program at CBM.

A sketch of the proposed configuration of the set-up of the experiment is shown in fig. 4. The experiment combines high precision track measurements with time-of-flight information for particle identification and total energy measurements for the analysis of the collision centrality. The charged track momentum and multiplicity will be measured with the set of twelve planes of GEM (Gaseous Electron Multipliers) detectors with two-coordinate readout located downstream of the target in the analyzing magnet and the drift/straw chambers (DCH, Straw) situated outside the magnetic field. The GEM detectors sustain high rates of particles and are operational in the strong magnetic field. The gap between the poles of the analyzing magnet is around $1 \mathrm{~m}$. The magnetic field can be varied up to $1.2 \mathrm{~T}$ to get the optimal BM@N detector acceptance and momentum resolution for different processes and beam energies. The available drift chambers are suited for reconstruction of interactions of light and medium ion beams. The straw tube detectors will be constructed in addition to the drift chambers to increase the reconstruction efficiency of the outer tracker for heavy ion beams. The design parameters of the time-of-flight detectors based on multi-gap resistive plate chambers (mRPC$1,2)$ with a strip read-out allow us to discriminate between hadrons $(\pi, \mathrm{K}, \mathrm{p})$ as well as light nuclei with the momentum up to few $\mathrm{GeV} / \mathrm{c}$ produced in multi-particle events. The zero degree calorimeter (ZDC) is designed for the analysis of the collision centrality by measuring the energy of forward going particles. The T0 detector, partially covering the backward hemisphere around the target, is planned 
Table 1. Beam parameters and setup at different stages of the experiment

\begin{tabular}{llllll}
\hline year 2016 & $\begin{array}{l}2017 \\
\text { spring }\end{array}$ & $\begin{array}{l}2017 \\
\text { autumn }\end{array}$ & 2020 & $\begin{array}{l}2021 \\
\text { and later }\end{array}$ \\
\hline beam $\mathrm{d}(\uparrow)$ & $\mathrm{C}, \mathrm{Ar}$ & $\mathrm{Kr}$ & $\mathrm{Au}$ & $\mathrm{Au}, \mathrm{p}$ \\
$\begin{array}{l}\text { maxim. } \\
\text { intensity, }\end{array}$ & $0.5 \mathrm{M}$ & $0.5 \mathrm{M}$ & $1 \mathrm{M}$ & $10 \mathrm{M}$ \\
$\begin{array}{l}\mathrm{Hz} \\
\text { trigger } 5 \mathrm{k}\end{array}$ & $5 \mathrm{k}$ & $5 \mathrm{k}$ & $10 \mathrm{k}$ & $20 \rightarrow 50 \mathrm{k}$ \\
$\begin{array}{l}\text { rate, Hz } \\
\text { central 6 GEM }\end{array}$ & $6 \mathrm{GEM}$ & $6 \mathrm{GEM}$ & $8 \mathrm{GEM}$ & $8 \mathrm{GEMs}+$ \\
$\begin{array}{l}\text { tracker half pl. } \\
\text { status }\end{array}$ & half pl. & half pl. & full pl. & Si planes \\
$\begin{array}{l}\text { experim.techn. } \\
\text { status run }\end{array}$ & $\begin{array}{l}\text { techn. } \\
\text { run }\end{array}$ & $\begin{array}{l}\text { techn. } \\
\text { run }\end{array}$ & $\begin{array}{l}\text { stage 1 } \\
\text { physics }\end{array}$ & $\begin{array}{l}\text { stage 2 } \\
\text { physics }\end{array}$ \\
\hline
\end{tabular}

to trigger central heavy ion collisions and provide a start time (T0) signal for the mRPC-1,2 detectors. An electro-magnetic calorimeter will be installed behind the mRPC-2 wall to study processes with electro-magnetic probes $\left(\gamma, e^{ \pm}\right)$in the final state.

\section{BM@N simulations and feasibility study}

Activities on the detector and beam line construction are complemented with intensive Monte Carlo simulation studies for optimization of the detector set-up. Special focus is set on the measurement of strange hyperons and hyper-nuclei in $\mathrm{Au}+\mathrm{Au}$ collisions at the maximal kinetic beam energy of 4.5 AGeV. The simulation of Au+Au collisions is performed using the URQMD [18] and DCMQGSM [19] models for heavy ion collisions. The products of collisions are transported through the BM@N setup using the GEANT program and reconstructed using the track reconstruction algorithm for multi-particle events [20].

Figure 5 illustrates the distribution of primary protons generated in $\mathrm{Au}+\mathrm{Au}$ collisions at the kinetic beam energy of $4.5 \mathrm{AGeV}$ in the phase space of the transverse momentum and rapidity in the laboratory frame. The product of the geometrical acceptance and track reconstruction efficiency of the GEM tracker for primary protons for the same phase space is shown on the lower plot. Figure 6 presents the momentum resolution and the vertex impact parameter resolution of charged particles reconstructed in the GEM tracker. The results are presented for the magnetic field in the center of the magnet of $0.44 \mathrm{~T}$. The simulation results indicate that the proposed set-up has a reasonable reconstruction capability for strange hyperons produced in high multiplicity central $\mathrm{Au}+\mathrm{Au}$ collisions [23]. The expected statistics is sufficient to perform measurements of strange hyperon and hyper-nuclei production yields and ratios, transverse momentum spectra, rapidity and angular distributions [24, 25]. Studies of fluctuations of event properties and correlations between products of nucleus-nucleus interactions are also feasible. 


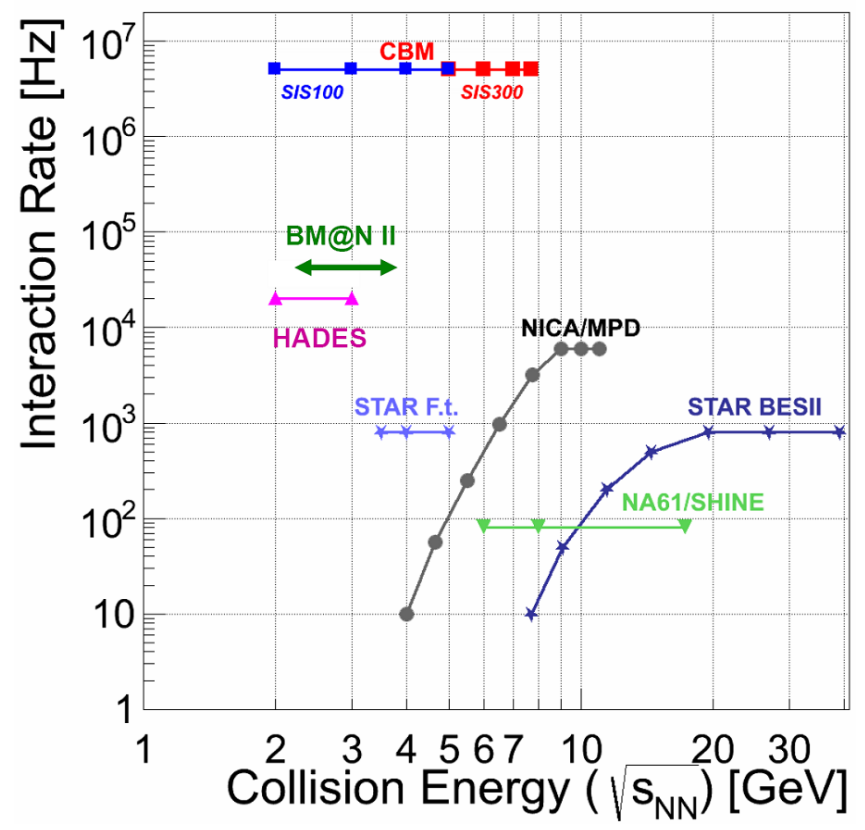

Figure 3. Heavy ion experiments: interaction rate and nucleon-nucleon collision energy in c.m.s. The BM@N range is superimposed on the plot taken from [16].

\section{Technical runs with the deuteron and carbon beams}

The technical runs of the BM@N detector were performed with the deuteron beam in December 2016 and with the carbon beam in March 2017. The schematic view of the BM@N setup used in the runs is presented in fig. 7. The view of the BM@N detectors behind the analyzing magnet and the GEM detectors inside the analyzing magnet are shown in fig. 8 . The kinetic energy was $4 \mathrm{GeV} /$ nucleon for the deuteron beam and varied from 3.5 to $4.5 \mathrm{GeV}$ for the carbon beam. The experimental data from the GEM detectors, forward silicon detector, drift chambers, time-of-flight detectors, zero degree calorimeter and trigger detectors were read out using the integrated data acquisition system. The starting configuration of the central tracker was based on 6 GEM stations combined from 5 GEM detectors with the size of $66 \times 41 \mathrm{~cm}^{2}$ and 2 GEM detectors with the size of $163 \times 45 \mathrm{~cm}^{2}$. Since the amount of the detectors was quite limited and one of the tasks was to measure the beam momentum, the tracking stations were arranged to have the beam passing through their centers. The detailed description of the GEM tracking system is done in [21, 22].

The main goal of the technical runs was the commissioning of the central GEM tracker and forward silicon detector and testing and calibration of the other subsystems: Time-of-Flight, trigger detectors, ZDC and ECAL calorimeters. The methodical goal of the runs was to trace the primary 


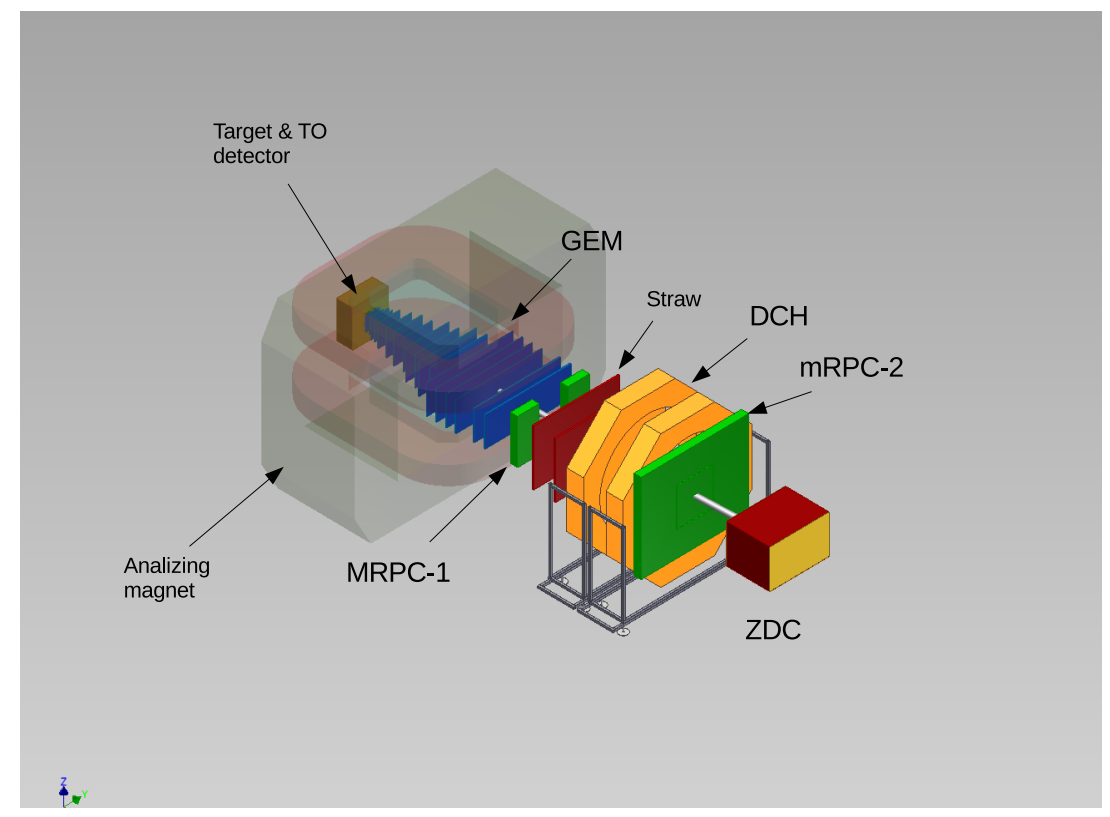

Figure 4. Schematic view of the BM@N setup.
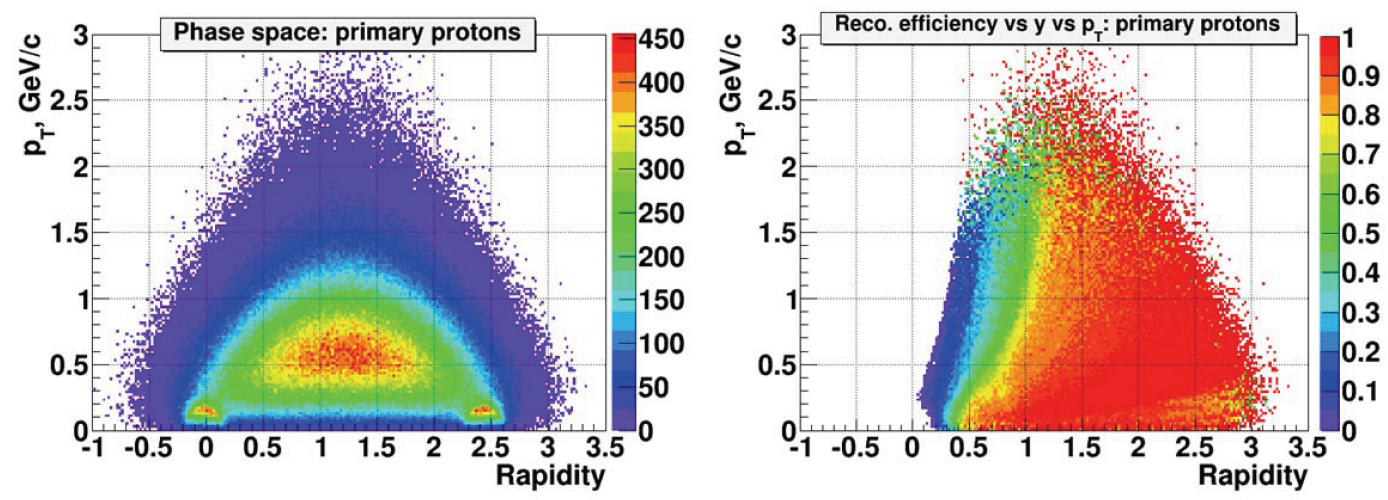

Figure 5. Left plot: distribution of primary protons generated in $\mathrm{Au}+\mathrm{Au}$ collisions at the kinetic beam energy of $4.5 \mathrm{AGeV}$ in the phase space of the transverse momentum and rapidity in the laboratory frame. Right plot: product of the geometrical acceptance and track reconstruction efficiency in the GEM tracker for primary protons as a function of the particle transverse momentum and rapidity. 

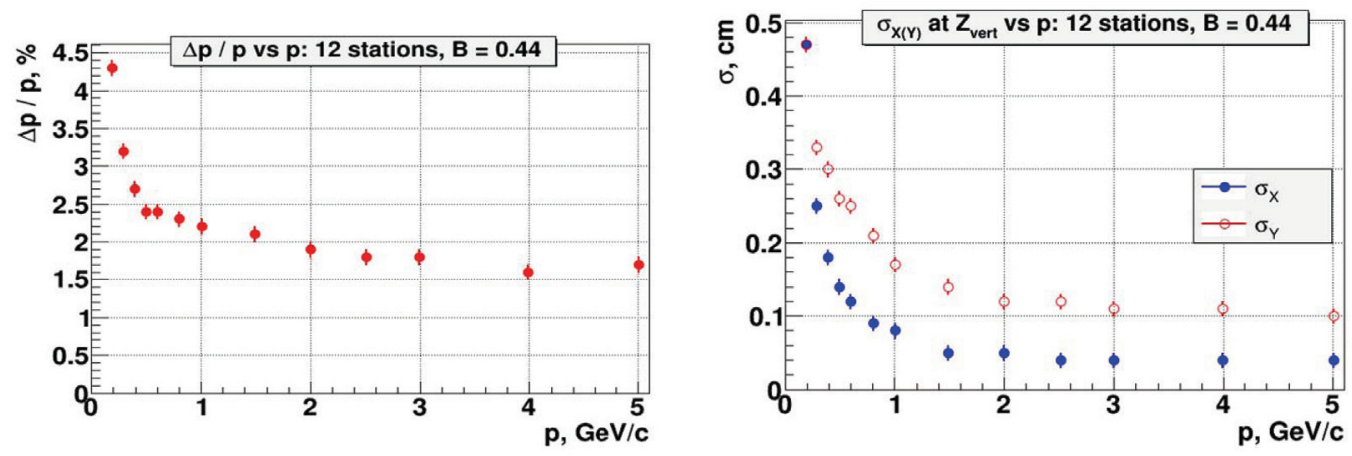

Figure 6. Momentum resolution (left plot) and vertex impact parameter resolution (right plot) of charged particles reconstructed in the GEM tracker shown as a function of the particle momentum.

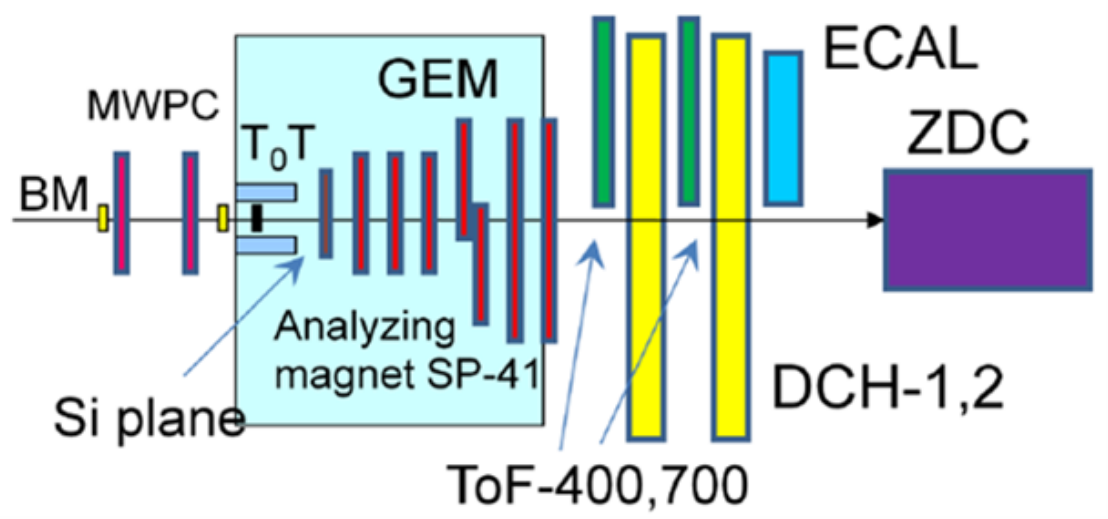

Figure 7. Schematic view of the BM@N set-up used in the deuteron and carbon runs.

beam through the set-up with subsequent reconstruction of its momentum at different values of the magnetic field.

The reasearch program was devoted to measurements of inelastic reactions $d, C+$ target $\rightarrow X$ and with the beam kinetic energy of 3.5, 4 and $4.5 \mathrm{~A} \mathrm{GeV}$ and different targets: $\mathrm{C}_{2} \mathrm{H}_{4}, \mathrm{C}, \mathrm{Al}, \mathrm{Cu}$. Since the GEM tracker configuration was tuned to measure relatively high-momentum beam particles, the geometric acceptance for relatively soft decay products of strange V0 particles was rather low. The Monte Carlo simulation showed that only $\sim 4 \%$ of $\Lambda$ hyperons and $\sim 0.8 \%$ of $K_{s}^{0}$ could be reconstructed.

\section{First results of the technical runs}

$\Lambda$-hyperons were reconstructed using their decay mode into two oppositely-charged tracks. The signal event topology (decay of a relatively long-lived particle into two tracks) defined the selection criteria: relatively large distance of the closest approach $(D C A)$ to the primary vertex of decay products, small track-to-track separation in the decay vertex, relatively large decay length of the mother particle. Since particle identification at this stage of the analysis was not used, all positive tracks were considered as protons and all negative as $\pi^{-}$. The invariant mass distributions of $p$ and $\pi^{-}$are shown in fig. 9 for reconstructed interactions of the deuteron and carbon beams with the targets. 

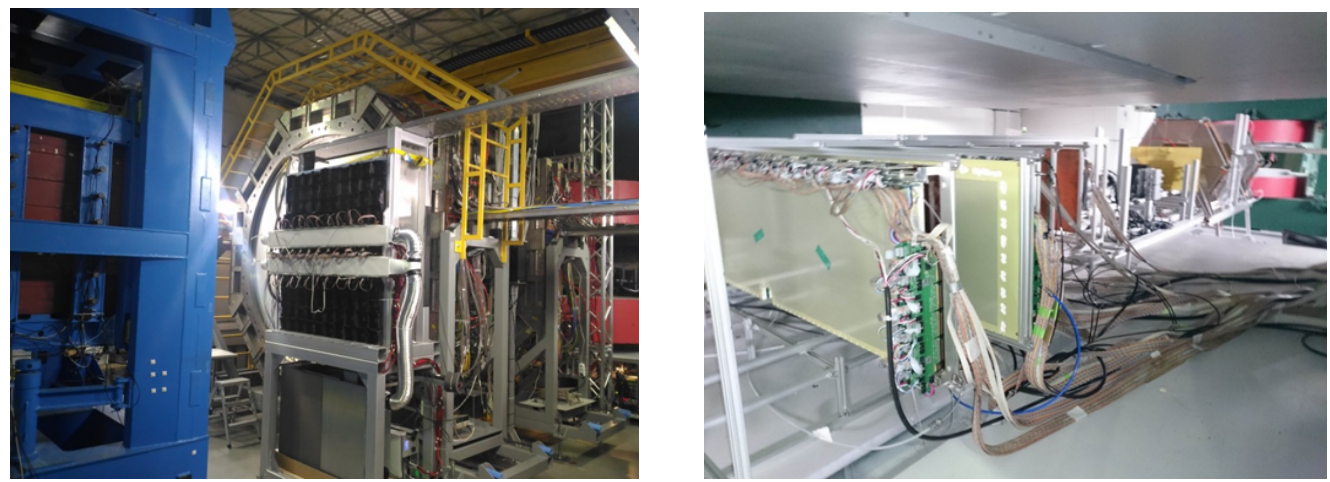

Figure 8. Left plot: BM@N setup behind the analyzing magnet in the technical runs. Right plot: GEM detectors inside the analyzing magnet.
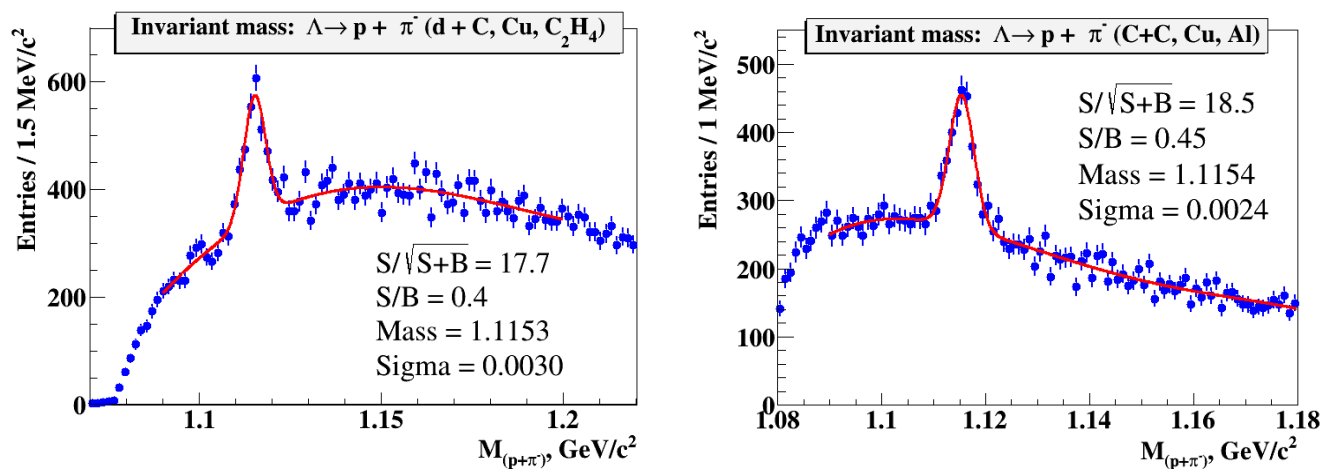

Figure 9. Invariant mass spectrum of pairs of protons and $\pi^{-}$reconstructed in interactions of the deuteron beam (left plot) and carbon beam (right plot) with the targets.

\section{Summary and plans}

The scientific program of the BM@N experiment comprises studies of nuclear matter in intermediate energy range between experiments at SIS and NICA/FAIR facilities. The BM@N experiment is in the starting phase of its operation and has recorded first experimental data. The technical runs were performed in the deuteron and carbon beams with the kinetic energy from 3.5 to $4.5 \mathrm{GeV}$ per nucleon. The collected data are used to check efficiencies of sub-detectors and develop algorithms for event reconstruction and analysis. In particular, experimental data of minimum bias interactions of the beam with different targets were analyzed with the aim to reconstruct tracks, primary and secondary vertexes using the central tracking detectors. The signal of $\Lambda$-hyperon was reconstructed in the invariant mass spectrum of particles originated from secondary vertexes. The extended configuration of the BM@N set-up is being realized for the next run with the argon and krypton beams. For future studies of $\mathrm{Au}+\mathrm{Au}$ collisions the collaboration with the CBM experiment is foreseen to produce and install large aperture STS silicon detectors in front of the GEM tracking detectors. 


\section{References}

[1] NICA White Paper, http://theorQ.jinr.ru/twiki-cgi/view/NICA/NICAWhitePaper

[2] B. Friman, W. Nörenberg, and V.D. Toneev, Eur. Phys. J. A 3 (1998).

[3] R. Rapp, J. Wambach, Eur. Phys. J. A 6, 415 (1999) .

[4] G. E. Brown, Prog. Theor. Phys. 91, 85 (1987).

[5] W. Cassing and E. L. Bratkovskaya, Phys. Rep. 308, 65 (1999).

[6] J. Steinheimer et al., Progress in Particle and Nuclear Physics 62, 313317 (2009).

[7] J. Steinheimer, K. Gudima, A. Botvina, I. Mishustin, M. Bleicher, H. Stocker, Phys. Lett. B 714, 85 (2012).

[8] C. Blume, J. Phys. G 31, S57 (2005) .

[9] A. Andronic et al., Phys. Lett. B 695, 203 (2011).

[10] BM@N Conceptual Design Report. http://nica.jinr.ru/files/BM@N/BMN_CDR.pdf

[11] G. Trubnikov, A. Kovalenko, V. Kekelidze, I. Meshkov, R. Lednicky, A. Sissakian, A. Sorin, PoS, ICHEP 2010, July 22-28, Paris, France, p.523.

[12] G. Trubnikov, N. Agapov, V.Kekelidze, A. Kovalenko, V. Matveev, I.Meshkov, R. Lednicky, A. Sorin, PoS, ICHEP 2012, July 4-11, Melbourne, Australia, p.411.

[13] V. Kekelidze, A. Kovalenko, R. Lednicky, V. Matveev, I.Meshkov, A. Sorin, G. Trubnikov, PoS, ICHEP 2014, July 2-9, Valencia, Spain.

[14] V.Golovatyuk, V.Kekelidze, V.Kolesnikov, O.Rogachevsky, A.Sorin, Eur.Phys.J. A52 (2016) no.8, 212

[15] V. Golovatyuk, M. Kapishin, V. Kekelidze, V. Kolesnikov, O. Rogachevky and A. Sorin, J.Phys.Conf.Ser. 668 (2016) no.1, 012015

[16] V. Friese, Strangeness Prospects with the CBM Experiment, talk at the SQM-2015 Conference, Dubna, Russia, July 6-11, 2015.

[17] J. Heuser et al., Technical Design Report for the CBM Silicon Tracking System, CBM Collaboration, GSI Report 2013-4, http://repository.gsi.de/record/54798

[18] URQMD model, http://urqmd.org/

[19] DCM-QGSM model, A.S. Botvina et al., Rhys. Rev. C 84, 064904 (2011).

[20] I. Kisel, Nucl. Instrum. Meth. A 566, 85 (2006).

[21] D.Baranov et al., GEM tracking system of the BM@N experiment, JINST 12 (2017) no.06, C06041

[22] BM@N Technical Design Report for the GEM Tracking System.

http://bmnshift.jinr.ru/wiki/lib/exe/fetch.php?media=tdr_gem_may2017_v1.doc

[23] D. Suvarieva, M. Ilieva, M. Kapishin, V. Kolesnikov, V. Vasendina and A. Zinchenko, J.Phys.Conf.Ser. 668 (2016) no.1, 012121

[24] M.Kapishin (for the BM@N Collaboration), Eur.Phys.J. A52 (2016) no.8, 213

[25] M.Kapishin (for the BM@N Collaboration), Phys. of Atom. Nuclei (2017) vol.8, no.11 